\title{
Patient experience and utility of genetic information: a cross- sectional study among patients tested for cancer susceptibility and thrombophilia
}

\author{
Elvira D'Andrea $\mathbb{(}^{1,2} \cdot$ Tyra Lagerberg $^{3} \cdot$ Corrado De Vito $^{1} \cdot$ Erica Pitini $\mathbb{D}^{1} \cdot$ Carolina Marzuillo $^{1} \cdot$ Azzurra Massimi $^{1}$. \\ Maria Rosaria Vacchio ${ }^{1}$. Paola Grammatico ${ }^{4} \cdot$ Paolo Villari $^{1}$
}

Received: 11 August 2017 / Revised: 13 November 2017 / Accepted: 5 December 2017

(c) The Author(s) 2018. This article is published with open access

\begin{abstract}
We evaluated whether genetic tests with evidence of clinical and personal utility (i.e. APC and BRCA1/2 tests) are associated with higher satisfaction and a more positive perception of care experience than those with undefined utility (i.e. tests for thrombophilia). A cross-sectional survey was performed through telephone interviews to patients tested for deleterious variants in $A P C$ or BRCA1/2 genes, or for inherited thrombophilia (FV Leiden and/or FIIG20210A) during a 5-year period (2008-2012). Three aspects of patient experience were assessed: effective communication through pre- and post-test genetic counselling; collaboration between caregivers on the management of patient care; and impact of genetic testing on quality of life. Overall 237 patients had telephone interviews. Multivariate logistic regression analyses showed that patients tested for $A P C$ or $B R C A 1 / 2$ variants were more likely to be satisfied with both pre- and post-test counselling than those tested for inherited thrombophilia (APC vs. thrombophilia, $p=0.039$ and $0.005 ; B R C A 1 / 2$ vs. thrombophilia, $p=0.030$ and $<0.001$ ). Patients tested for $A P C$ were more likely to report an improvement in quality of life than those for thrombophilia $(\mathrm{OR}=$ $2.97,95 \%$ CI $1.14,7.72 ; p=0.025)$. A positive association was observed between patients who underwent $B R C A 1 / 2$ testing, and self-perceived improvement in quality of life $(\mathrm{OR}=1.41,95 \% \mathrm{CI} 0.74,2.69 ; p=0.294)$. Tests of undefined clinical and personal utility are associated with a lower degree of patient satisfaction with genetic counselling and no clear opinions on changes in quality of life compared with those with well-defined utility.
\end{abstract}

\section{Introduction}

Over the last decade, researchers and policy makers have made measuring and improving the patient experience of genetic health-care services a high priority $[1,2]$. Although focusing on patient experience has been controversial, the bulk of the evidence suggests that high performance in these

Elvira D'Andrea

eld716@mail.harvard.edu

1 Department of Public Health and Infectious Diseases, Sapienza University of Rome, Rome, Italy

2 Brigham and Women's Hospital, Harvard Medical School, Boston, USA

3 Better Value HealthCare, Ltd, Oxford, UK

4 Laboratory of Medical Genetics, Department of Molecular Medicine, San Camillo-Forlanini Hospital, Sapienza University, Rome, Italy measures is associated with high performance in other aspects of health-care quality, such as clinical processes, patient adherence to prevention and treatment measures, and even health outcomes, particularly of chronic conditions [3-6]. Consequently, as for other health-care services, patient satisfaction and perception of their health-care experiences are expected to be increasingly used as a measure of performance in public reporting and programmes related to genetic testing $[2,6]$.

Patient evaluation of health-care services is usually based on features such as the interpersonal and communication skills of caregivers, the responsiveness of clinical staff to patient needs, and dialogue between different providers in the clinical process [6]. In the case of predictive genomic applications, such features are complicated by the value-or overall utility - of the genetic information obtained. Overall utility includes the evaluation of clinical measures, such as decreased morbidity and mortality, pursuit of prophylactic treatments or follow-up surveillance, and health-related quality of life $[7,8]$, as well as personal or social outcomes 
that include subjective, non-health-related uses of genetic information such as improving feelings of control, enhancing self-knowledge, and planning for the future $[8,9]$. The lack of evidence on personal and/or clinical utility of genetic testing can make it difficult to lead physicians in clinical decision-making and to provide patients with appropriate information, which can therefore have a negative impact on patient experience.

In this study, we evaluated whether genetic tests with evidence of clinical and personal utility are associated with higher satisfaction and a more positive perception of the care experience than genetic tests with undefined utility. As genetic tests with well-defined utility, we included testing for dominant germline variants in the adenomatous polyposis coli (APC) gene, which have almost $100 \%$ penetrance and cause about $1 \%$ of all colorectal cancers [10], and testing for dominant variants in one of the breast/ ovarian cancer susceptibility genes (BRCA1, BRCA2), which have $40-70 \%$ penetrance and are responsible for $2-7 \%$ of breast cancers and $10-15 \%$ of ovarian cancers [11]. Genetic testing for two variants (Factor V Leiden and/or FIIG20210A) of inherited thrombophilia, which is associated with low-risk susceptibility to venous thromboembolism (VTE), was selected as a genetic test with undefined utility [12]. Three aspects of patient satisfaction and experience were assessed: effective communication through pre- and post-test genetic counselling; collaboration between health-care providers on the management of patient care; and impact of genetic testing on quality of life.

\section{Methods}

\section{Study population}

Participants were recruited from the genetic service of the San Camillo-Forlanini Hospital (Rome, Italy). Eligibility criteria were that patients were tested for deleterious variants in $A P C$ or $B R C A 1 / 2$, or for inherited thrombophilia (Factor V Leiden and/or FIIG20210A) during a 5-year period (2008-2012), and that they obtained the results of these tests. Patients were excluded if they had prior or ongoing major psychiatric dysfunction or neurological disease. All individuals selected received an advance pack including an informed consent form and a cover letter that outlined the details of the study and informed patients of their guaranteed anonymity. Individuals who agreed to participate completed the informed consent forms and returned them to the genetic service. All telephone interviews were administered by three researchers (ED, EP, MRV) between November 2013 and March 2014. The study was approved by the ethics committee of San Camillo-Forlanini Hospital.

\section{Survey instrument}

The set of questions assessed in this study was part of a wider survey designed to investigate the appropriateness of genetic testing delivery and post-testing health-care pathways. The questions evaluate patient socio-demographic and professional characteristics; the familial, clinical, and genetic characteristics of patients (known variant in the family, carrier status, previous primary cancer or thrombotic event, etc.); and the patients' experience of the genetic testing process. Three self-reported elements were used to measure patient experience: patient satisfaction with genetic counselling, patient perception of collaboration between caregivers both within and outside the genetic service, and the impact of genetic testing on patient quality of life.

We posed four questions relating to genetic counselling: two on whether or not pre- and post-test genetic counselling was performed, and two on patient satisfaction with counselling, before and after testing, using a five-point Likert scale ('very satisfied', 'satisfied', 'uncertain', 'dissatisfied', 'very dissatisfied'). Collaboration between health-care actors (as perceived by the patient) was explored through three questions. The first two asked about the kind of physician who referred the patients for testing, and whether the genetic counsellors suggested health-care centres or a specialized team of experts for the management of specific hereditary disorders. The third question investigated patient perception of the collaboration among physicians (specifically clinical geneticists and specialists) through a five-point Likert scale ('definitely yes', 'probably yes', 'uncertain', 'probably no', 'definitely no'). In two final questions, participants were asked whether performing the genetic testing had improved or damaged their quality of life, using a fivepoint Likert scale ('definitely yes', 'probably yes', 'uncertain', 'probably no', 'definitely no').

An extensive pilot phase was conducted with a convenience sample of 30 participants (ten for each type of genetic test) to ensure practicability of the survey, as well as validity and appropriate interpretation of the survey outcomes. On the basis of the comments and suggestions obtained from the pilot study, the questionnaire was revised before distribution to the study sample. Items were only included in the survey instrument if there was consensus on their meaning among participants. A clear need to slim down the questionnaire emerged. Therefore, we decided not to adopt a validated questionnaire and instead to ask about the quality of life, including any damage suffered, using two direct questions. Because the survey was administered by telephone, we attempted to explain the meaning of questions to participants. 


\section{Statistical analyses}

The demographic and clinical characteristics of the study participants are stratified by type of genetic test. Standard deviations are presented for continuous variables, and relative frequencies for categorical ones. The outcome variables, originally consisting of five categories, were collapsed into two levels. Patients were considered satisfied if they rated their satisfaction as 'very satisfied' or 'satisfied'. Perception of collaboration between providers, selfperceived improvement of quality of life and selfperceived damage to health were considered positive if patients answered 'definitely yes' or 'probably yes'.

Multivariate logistic regression analyses were used to identify the association between the type of genetic testing and (i) patient satisfaction with pre-test and post-test genetic counselling (models 1 and 2); (ii) perceived collaboration between caregivers in the genetic testing pathway (model 3); (iii) perceived improvement in quality of life (model 4). All multivariate models included carrier status as a dichotomous variable (carriers of $A P C, B R C A 1 /$ 2, Factor V Leiden and/or FIIG20210A variants vs. noncarriers), because carrier status is a potential confounder of associations between type of test and patient experience (satisfaction, perception of adequate collaboration and quality of life). These associations were assessed by the purposeful selection method [13]. Any variable having a significant univariate analysis at $p$-value $(p)<0.25$ for an association with the outcome of interest was selected as a candidate for the multivariate analysis. Covariates were removed from the model if they were non-significant at alpha 0.1 and not a confounder, defined as a covariate whose removal caused a change in the main effect of the remaining parameter estimates greater than 20\%. Any variables not selected for the original model were added back one at a time, to identify those that make an important contribution in the presence of other variables. After repeating the process of deleting, refitting and verifying, the models contained significant covariates and confounders. Collinear variables were eliminated in the process. In model 1 , we detected a quasi-complete separation of the data points of the variable testing type (dummy APC testing). Exact logistic regression was performed to solve this issue [14]. Median unbiased estimates, 95\% CI and exact $p$-value were reported for dummy APC testing in model 1 . In model 4, we added interaction terms to investigate if carrier status is an effectmeasure modifier of the association between the tests and self-perceived improvement in quality of life.

STATA version 14.0 was used for all calculations (College Station, Texas, Stata Corporation, 2015). Throughout the paper, $p<0.05$ is regarded as significant.

\section{Results}

During the study period, 370 eligible patients were approached. Of these, 237 (64\%) agreed to participate and completed the questionnaires. Patients tested for $A P C$ or $B R C A 1 / 2$ variants were more likely to complete the survey than those tested for thrombophilia (80\% [33/41], 78\% [104/133] vs. 51\% [100/196], $p<0.01)$. Eligible patients who did not complete the questionnaires were comparable to those who were interviewed (age, gender and level of education; $p>0.05$ ).

\section{Sample characteristics}

Of the 237 patients who had telephone interviews, 33 (14\%) had been tested for $A P C, 104$ (44\%) for BRCA1/2 and 100 $(42 \%)$ for thrombophilia (Factor $\mathrm{V}$ Leiden and/or FIIG20210A) variants (Table 1). On average, patients tested for $A P C$ variants were younger than those tested for $B R C A 1 / 2$ or thrombophilia variants [mean age $36( \pm 20)$ years vs. $52( \pm 14)$ and $46( \pm 14), p=0.203]$. The percentage of female respondents ranged from $87 \%$ for $B R C A 1 / 2$ questionnaires to $51 \%$ for $A P C$ questionnaires $(p<0.01$ across groups). Overall, $77 \%$ of the sample completed at least high school (45\% intermediate and 32\% a high level of education), and the level of education was similar across the groups (Table 1). More than half of the respondents for the $A P C \quad(58 \%)$ and thrombophilia questionnaires $(56 \%)$ reported as a carrier of a deleterious variant, while $71 \%$ of BRCA1/2 respondents were not carriers $(p<0.01$ across groups). Six individuals tested for thrombophilia did not recall having had a genetic test and were not aware of their carrier status. Information about family history and known familial variants varied across the different types of genetic testing $(p<0.01)$. Sixty-one per cent of patients who underwent $A P C$ testing indicated a positive family history and a known deleterious variant in their families. Patients were mainly referred for $B R C A$ testing because of a positive family history, but where the familial variants were unknown (51\%). Half of the patients tested for thrombophilia reported no family history and no known variants in their families (Table 1).

\section{Patient satisfaction with pre- and post-test counselling}

Ninety-seven and $98 \%$ of all respondents tested for $A P C$ and $B R C A$ variants, respectively, received appropriate information before testing, vs. $72 \%$ of patients tested for thrombophilia $(p<0.01)$ (Table 2). Of all patients who received pre-testing information, $94 \%$ were satisfied with their understanding of the testing procedure, the benefits and limitations of the test, and the possible consequences of 
Table 1 Demographic and clinical characteristics of patients who underwent genetic testing (APC, BRCA or thrombophilia) at the genetic service of the San CamilloForlanini Hospital

\begin{tabular}{|c|c|c|c|c|c|}
\hline \multirow[t]{2}{*}{ Variables } & \multicolumn{4}{|c|}{ Testing type } & \multirow[b]{2}{*}{$p$-value } \\
\hline & $A P C$ & BRCA1/2 & Thrombophilia & Total & \\
\hline Patients, $n(\%)$ & $33(14 \%)^{\mathrm{a}}$ & $104(44 \%)$ & $100(42 \%)$ & 237 & \\
\hline Age, mean (SD), yy & $36( \pm 20)$ & $52( \pm 14)$ & $46( \pm 14)$ & $47( \pm 16)$ & 0.203 \\
\hline Female, $n(\%)$ & $17(51 \%)$ & $91(87 \%)$ & $69(69 \%)$ & $177(75 \%)$ & $<0.001$ \\
\hline \multicolumn{6}{|l|}{ Level of education, $\mathrm{n}(\%)^{b}$} \\
\hline Low & $8(24 \%)$ & $28(27 \%)$ & $19(19 \%)$ & $55(23 \%)$ & \multirow[t]{3}{*}{0.480} \\
\hline Intermediate & $17(52 \%)$ & $41(39 \%)$ & $49(49 \%)$ & $107(45 \%)$ & \\
\hline High & $8(24 \%)$ & $35(34 \%)$ & $32(32 \%)$ & $75(32 \%)$ & \\
\hline \multicolumn{6}{|c|}{ Deleterious variant carriers, $\mathrm{n}(\%)$ [missing 6] } \\
\hline Carriers & $19(58 \%)$ & $30(29 \%)$ & $53(56 \%)$ & $102(44 \%)$ & \multirow[t]{3}{*}{$<0.001$} \\
\hline Non-carriers & $11(33 \%)$ & $74(71 \%)$ & $27(29 \%)$ & $112(48 \%)$ & \\
\hline Carriers of other variants ${ }^{\mathrm{c}}$ & $3(9 \%)$ & - & $14(15 \%)$ & $17(7 \%)$ & \\
\hline \multicolumn{6}{|c|}{ Family history $(\mathrm{FH})$ and known familial variant $(\mathrm{KFV}), \mathrm{n}(\%)$} \\
\hline$F H-$ and $K F V-$ & $5(15 \%)$ & $16(15 \%)$ & $50(50 \%)$ & $71(30 \%)$ & \multirow[t]{4}{*}{$<0.001$} \\
\hline$F H+$ and $K F V-$ & $8(24 \%)$ & $53(51 \%)$ & $24(24 \%)$ & $85(36 \%)$ & \\
\hline$F H-$ and $K F V$ & - & - & $6(6 \%)$ & $6(2.53 \%)$ & \\
\hline$F H+$ and $K F V+$ & $20(61 \%)$ & $35(34 \%)$ & $20(20 \%)$ & $75(32 \%)$ & \\
\hline Testing-interview time, mean (SD), yy & $4( \pm 2)$ & $3( \pm 1)$ & $3( \pm 1)$ & $3( \pm 1)$ & $<0.001$ \\
\hline
\end{tabular}

Low Primary school, lower level of secondary school; Intermediate higher level of secondary school; High university, master degree, specialization

${ }^{a}$ Including eight minors

${ }^{\mathrm{b}}$ In the case of minors, we counted the highest education level among the parents

c Three patients tested for $A P C$ variants were carriers of an MUTYH variant; 14 patients tested for thrombophilia were carriers of an MTHFR variant the results. Patients tested for $A P C$ and $B R C A$ were more satisfied than those tested for thrombophilia (100\% and $98 \%$ vs. $86 \%, p=0.002)$. Geneticists play a central role in post-test genetic counselling for APC $(67 \%)$ and BRCA1/2 testing (54\%), while the majority of patients tested for thrombophilia received information about test results and their implications from specialists (52\%). Few patients interviewed had integrated counselling by both a geneticist and a clinical specialist (Table 2). Of all patients who had been informed about their results, respondents who received genetic counselling for $A P C$ and $B R C A 1 / 2$ testing were more satisfied than those for thrombophilia testing $(p<$ $0.01)$.

\section{Patient experience of integrated care management}

Specialists played a central role in detecting clinical evidence of potential hereditary diseases and referring patients for related genetic testing (Table 2). Specifically, gastroenterologists were the specialists that more often referred patients for genetic counselling following APC testing (12 out of $16,75 \%$ ), oncologists for genetic counselling following BRCA1/2 testing (57 out of $67,85 \%$ ), and gynaecologists for thrombophilia testing (37 out of 94, 39\%). Few patients reported being referred for genetic tests by primary care physicians. Overall, very few patients received information, during counselling, about dedicated health-care centres (such as centres for rare diseases) or specialized teams of physicians who are particularly experienced in managing clinical cases like theirs. The majority of patients referred to a lack of communication or collaboration between clinical geneticists and other providers, regardless of the type of test (Table 2).

\section{Self-perception by patients of quality of life}

Improvements in the quality of life were more likely to be reported by patients who underwent $A P C$ and $B R C A 1 / 2$ testing than by those who underwent thrombophilia tests ( $p$ $=0.030)$. Almost none of the respondents perceived any damage to their lives due to the genetic examinations (Table 2).

\section{Multivariate analyses}

Table 3 shows adjusted analyses of patient satisfaction with pre- and post-test genetic counselling (models 1 and 2), perceived collaboration between caregivers in the genetic 
Table 2 Self-reported results on experience and satisfaction by patients who underwent genetic testing (APC, BRCA or thrombophilia) at the genetic service of the San CamilloForlanini Hospital

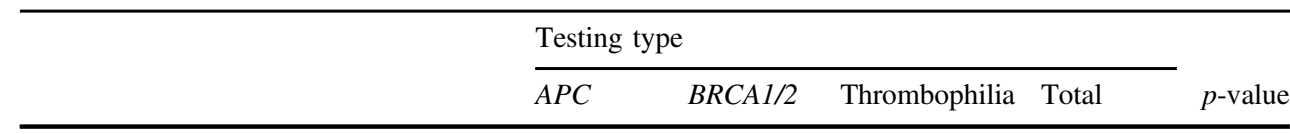

\section{Genetic counselling and testing procedure}

Q1. Before you had the genetic test, did you receive appropriate information about testing procedure, benefits and limitations of the test, and eventual results?

$\begin{array}{llllll}\text { Yes (face-to-face) } & 32(97 \%) & 102(98 \%) & 72(72 \%)^{\mathrm{a}} & 206(87 \%)^{\mathrm{a}}<0.001 \\ \text { No } & 1(3 \%) & 2(2 \%) & 28(28 \%) & 31(13 \%)\end{array}$

Q2. If yes, how satisfied are you with your understanding of the testing procedure, the benefits and limitations of the test, and the possible consequences of the test results?
Satisfied
$32(100 \%) \quad 100(98 \%) \quad 62(86 \%)$
$194(94 \%) \quad 0.002$
Uncertain or dissatisfied
$2(2 \%) \quad 10(14 \%)$
$12(6 \%)$

Q3. After you had the genetic test, did you receive genetic counselling? (1 missing)

$\begin{array}{llllll}\text { Yes, by a geneticist } & 22(67 \%) & 56(54 \%) & 16(16 \%) & 94(40 \%) & <0.001 \\ \text { Yes, by a specialist } & 2(6 \%) & 7(7 \%) & 51(52 \%) & 60(25 \%) & \\ \text { Yes, by both a geneticist and a specialist } & 6(18 \%) & 37(35 \%) & 14(14 \%) & 57(24 \%) \\ \text { No } & 3(9 \%) & 4(4 \%) & 18(18 \%) & 25(11 \%)\end{array}$

Q4. If yes, how satisfied are you with your understanding of what the test results mean, including your understanding of positive and negative results, and of the potential for uninformative results or incorrect results (such as false positives or false negatives)? (1 missing)

$\begin{array}{llllll}\text { Satisfied } & 29(97 \%) & 98(98 \%) & 49(60 \%) & 176(83 \%) & <0.001 \\ \text { Uncertain or dissatisfied } & 1(3 \%) & 2(2 \%) & 32(40 \%) & 35(17 \%) & \\ \begin{array}{l}\text { Integrated care management } \\ \text { Q5. Who referred you for genetic testing? }\end{array} & & & & & \\ \text { Primary care physician } & 5(15 \%) & 6(6 \%) & 6(6 \%) & 17(7 \%) & <0.001 \\ \text { Geneticist } & 12(36 \%) & 31(30 \%) & - & 43(18 \%) & \\ \text { Specialist } & 16(49 \%) & 67(64 \%) & 94(94 \%) & 177(75 \%)\end{array}$

Q6. If you have undergone genetic counselling, did the counsellor suggest dedicated health-care centres or specialized medical doctors who could manage your clinical case?

$\begin{array}{llllll}\text { Yes } & 5(15 \%) & 8(8 \%) & 13(13 \%) & 26(11 \%) & 0.440 \\ \text { No } & 28(85 \%) & 96(92 \%) & 87(87 \%) & 211(89 \%) & \end{array}$

Q7. Has there been collaboration between the health-care professionals who participated in your care (e.g. the clinicians who required testing and clinical geneticists who reported results, or clinical geneticists who performed the genetic counselling and clinicians who planned a surveillance programme, etc.)? (1 missing)
(Definitely or Probably) yes
$6(18 \%) \quad 19(18 \%) \quad 35(35 \%)$
$60(25 \%)$
0.014
Uncertain or (Definitely or Probably) no
$27(82 \%) \quad 85(82 \%) \quad 64(65 \%)$
$176(75 \%)$

Quality of life

Q8. Has the genetic testing improved your quality of life? ${ }^{b}$

\begin{tabular}{llllll} 
(Definitely or Probably) yes & $26(79 \%)$ & $77(74 \%)$ & $59(59 \%)$ & $162(68 \%)$ & 0.030 \\
Uncertain or (Definitely or Probably) no & $7(21 \%)$ & $27(26 \%)$ & $41(41 \%)$ & $75(32 \%)$ & \\
Q9. Has the genetic testing damaged your daily life? & & & \\
Uncertain or (Definitely or Probably) no & $33(100 \%)$ & $102(98 \%)$ & $100(100)$ & $235(99 \%)$ & 0.628 \\
(Definitely or Probably) yes & - & $2(2 \%)$ & - & $2(1 \%)$ & \\
\hline
\end{tabular}

\footnotetext{
${ }^{a}$ Three patients received both face-to-face and written information, and seven received only written information

${ }^{\mathrm{b}}$ Exploring quality of life included how patients were able to do certain things (energy for everyday life, acceptance of bodily appearance, opportunity for leisure activities and capacity of work) and how they experienced various aspects of their life (sleep, daily living activities, personal and social relationships)

${ }^{c}$ clinical, psychological and social (discrimination and stigmatization) harms were investigated.
}

testing pathway (model 3), and self-perceived improvement in life quality (model 4), across the three different types of genetic testing, assuming no bias is present. Patients tested for $A P C$ or $B R C A 1 / 2$ variants were more likely to be satisfied with pre-test counselling than those tested for inherited thrombophilia ( $A P C$ vs. thrombophilia, $p=0.039$; 
Table 3 Multivariate analyses of patient satisfaction with pre- and post-test genetic counselling (models 1 and 2), perceived collaboration between caregivers in the genetic testing pathway (model 3) and perceived improvement in quality of life by type of genetic test (model 4)

\begin{tabular}{lcll}
\hline & OR & $95 \%$ CI & p-value \\
\hline $\begin{array}{l}\text { Patient satisfaction with pre-test genetic counselling (model } 1) \\
\text { Type of genetic test }\end{array}$ & & & \\
APC test ${ }^{\text {a }}$ & $7.13^{\mathrm{a}}$ & $1.08-$ inf $^{\mathrm{a}}$ & $0.039^{\mathrm{a}}$ \\
BRCA1/2 tests & 6.00 & $1.19-30.36$ & 0.030 \\
Thrombophilia tests (reference) & 1.00 & - & - \\
Carrier status (reference $=$ non- & 0.44 & $0.11-1.81$ & 0.254 \\
carrier) & & &
\end{tabular}

Patient satisfaction with post-test genetic counselling (model 2)

Type of genetic testing

$\begin{array}{llll}\text { APC test } & 18.97 & 2.46-146.36 & 0.005 \\ \text { BRCA1/2 tests } & 32.44 & 7.07-148.88 & <0.001 \\ \text { Thrombophilia tests (reference) } & 1.00 & - & - \\ \text { Carrier status (reference }=\text { non- } & 1.03 & 0.41-2.58 & 0.948 \\ \text { carrier) } & & & \end{array}$

carrier)

Patient experience of collaboration between caregivers (model 3)

Type of genetic test

\begin{tabular}{llll} 
APC test & 0.28 & $0.08-1.08$ & 0.064 \\
BRCA1/2 tests & 0.49 & $0.17-1.37$ & 0.174 \\
Thrombophilia tests (reference) & 1.00 & - & - \\
Carrier status (reference = non- & 1.08 & $0.52-2.26$ & 0.830 \\
carrier) & \multicolumn{3}{l}{} \\
Type of health professional referring patient for test & \\
Geneticist & 0.43 & $0.11-1.71$ & 0.232 \\
Specialist & 0.22 & $0.07-0.77$ & 0.017 \\
Primary care physician (reference) & 1.00 & - & - \\
Post-test genetic counselling & & & \\
Yes, by a geneticist & 2.20 & $0.83-5.85$ & 0.113 \\
Yes, by a specialist & 0.55 & $0.19-1.58$ & 0.269 \\
Yes, by both a geneticist and a & 8.53 & $2.75-26.43$ & $<0.001$ \\
specialist & & & \\
No (reference) & 1.00 & - & - \\
Familiarity $($ reference $=$ no) & 2.39 & $1.02-5.60$ & 0.045
\end{tabular}

Self-perceived improvement in life quality (model 4 )

Type of genetic test

$\begin{array}{llll}\text { APC test } & 2.97 & 1.14-7.72 & 0.025 \\ \text { BRCA1/2 tests } & 1.41 & 0.74-2.69 & 0.294 \\ \text { Thrombophilia tests (reference) } & 1.00 & - & - \\ \begin{array}{l}\text { Carrier status (reference = non- } \\ \text { carrier) }\end{array} & 0.52 & 0.28-0.97 & 0.040 \\ \text { Gender (reference = male) } & 1.78 & 0.91-3.47 & 0.088\end{array}$

${ }^{a}$ Exact logistic regression was used because of convergence failure, median unbiased estimate, $95 \% \mathrm{CI}$ and exact $p$-value were reported for dummy APC testing [14].

$B R C A 1 / 2$ vs. thrombophilia, $p=0.030$ ). Furthermore, the odds of being satisfied with post-test counselling were higher in patients who underwent $A P C$ and $B R C A l / 2$ testing, respectively, than in patients with a history of thrombophilia testing ( $A P C$ vs. thrombophilia, $p=0.005$; $B R C A 1 / 2$ vs. thrombophilia, $p<0.01)$. In both models 1 and 2, carrier status does not seem to be a predictor of patient satisfaction with genetic counselling (pre-test counselling, odds ratio $(\mathrm{OR})=0.44,95 \%$ CI $0.11,1.81 ; p=$ 0.254 ; post-test counselling, $\mathrm{OR}=1.03,95 \%$ CI $0.41,2.58$; $p=0.948$ ).

Patients tested for inherited thrombophilia reported a more positive perception of collaboration between caregivers than those testing for cancer-related hereditary diseases (i.e. $A P C$ and $B R C A 1 / 2$ testing). Among patients with a history of $A P C$ and $B R C A$ testing, odds of adequate collaboration were respectively 72 and $51 \%$ lower than patients who underwent thrombophilia testing ( $A P C$ vs. thrombophilia, $\mathrm{OR}=0.28,95 \%$ CI $0.08,1.08 ; p=0.064$; OR $=$ $0.49, B R C A 1 / 2$ vs. thrombophilia, $95 \%$ CI $0.17,1.37 ; p=$ 0.174). Moreover, participants who received post-test counselling by both a geneticist and a specialist were more likely to report a positive collaboration between providers compared to those who did not receive counselling $(\mathrm{OR}=8.53,95 \%$ CI 2.75, 26.43; $p<0.01)$. Patients with positive familial history were more likely to report an adequate collaboration between different providers in the genetic pathway $(\mathrm{OR}=2.39,95 \%$ CI $1.02,5.60 ; p=$ 0.045).

Findings on the perception of quality of life were more favourable for cancer-related genetic tests than thrombophilia testing (Table 3). Patients who underwent APC testing were more likely to report an improvement in quality of life than those who underwent thrombophilia testing $(\mathrm{OR}=$ 2.97, 95\% CI 1.14, 7.72; $p=0.025)$. We also observed a positive association between patients tested for BRCAl/2 variants, and self-perceived improvement in quality of life $(\mathrm{OR}=1.41,95 \%$ CI 0.74, 2.69; $p=0.294)$. Carrier status was also significantly associated with improvement in quality of life ( $\mathrm{OR}=0.52,95 \% \mathrm{CI} 0.28,0.97 ; p=0.040)$, and this association did not change according to the type of test performed (interaction terms were not significant).

\section{Discussion}

The current study explored the relationship between patient experience (as represented by 'satisfaction with genetic counselling', 'perception of collaboration between caregivers' and 'self-perceived change in life quality') and different types of genetic test by interviewing patients who underwent testing at a genetic service. The genetic tests were selected on the basis of their clinical and personal utility. $A P C$ and $B R C A 1 / 2$ tests are strongly predictive with well-defined utility, and their results are widely utilized to lead clinical and personal decision-making $[10,15,16]$. By 
contrast, no significant clinical and personal benefits result from weakly predictive genetic tests such as that for Factor V Leiden and FIIG20210A [12, 17, 18]. In our study clinical utility was referred to the likelihood that the test results will lead to an improved health outcome, including healthrelated quality of life, pursuit of prophylactic interventions $[7,8]$; while personal utility takes into account the subjective, non-health-related usefulness of the test results (e.g., psychological, social and economic consequences) [8, 9]. Genetic tests with undefined utility can have a negative impact on patient experience and our results partially confirmed this thesis.

Our findings showed that the overall satisfaction with genetic counselling was high. Participants tested for thrombophilia were less likely to receive pre- or post-test genetic counselling. In some cases, they were informed only through a written document (Q1, Table 2). Furthermore, genetic counselling for thrombophilia tests was mainly performed by clinicians other than geneticists. These results reflect different adopted procedures. Strongly predictive genetic tests for life-threatening diseases-like those resulting from $A P C$ or $B R C A 1 / 2$ variants-were performed together with a genetic counselling service by clinical geneticists. By contrast, Factor V Leiden and FIIG20210A testing can take place either with or without genetic counselling; in both cases, information pre-test and results were communicated in written form.

On average, patients who underwent $A P C$ or $B R C A 1 / 2$ testing were more likely to report a higher degree of satisfaction with pre- and post-test genetic counselling than those tested for thrombophilia. A lower response rate was recorded among patients who underwent thrombophilia testing, which could imply that these patients were on average less sensitive to the topic of the survey, and therefore even less satisfied than what our results reported. One explanation for these results is that thrombophilia tests provide less valuable information than the other two tests. Patients value information such as likelihood of developing a condition due to genetic variants, timing of clinical onset in the life cycle, overall clinical severity and availability of effective interventions that can modify clinical onset and/or disease progression [19, 20]. Inherited thrombophilia is characterized by a low likelihood of developing VTE, which is generally a non-fatal disorder with an unpredictable timing of clinical onset and a lack of effective strategies for prevention $[12,17,18,21,22]$. This can be difficult for physicians to communicate effectively, which can cause confusion or distress among recipients, especially if the preventive strategies are limited [19]. A study by Saukko et al. [23] on thrombophilia testing reveals that lessinformed participants did not differ from well-informed ones in terms of adopted preventive measures. More broadly, there is a lack of clear evidence about the clinical and personal utility of thrombophilia testing, which can contribute to patient dissatisfaction with genetic counselling $[19,23]$.

We found no clear and meaningful evidence on the association between how patients perceived collaboration between caregivers in the genetic pathway and type of test. The direction of the effect suggested that patients who were tested for inherited thrombophilia were more likely to perceive care integration than those tested for $A P C$ or $B R C A 1 / 2$ variants, although the results were not significant. As expected, participants who received post-test genetic counselling by both a geneticist and a specialist were more likely to report a positive collaboration between providers than those who did not have counselling. International guidelines recommend that genetics services should be integrated with other clinical specialties, or, where there is existing integration, this should be improved [23]. Given that genetics services are undergoing expansion, the overall capacity of clinical geneticists is limited and other professionals need to be trained to support the appropriate change from compartmental services to integrated care management $[24,25]$.

It is interesting that none of the 100 interviewed patients was referred for thrombophilia testing by geneticists. More than one-third of the participants were referred by gynaecologists to inform the clinical decision on prescribing a combined oral contraceptive or hormone replacement therapy (HRT), or to support their advice to family members on these issues. This finding is consistent with the current literature [23], which describes prescription of oral contraceptives or HRT as the main reason for thrombophilia testing. Several studies have reported that most referrals for thrombophilia tests by clinicians were not appropriate and did not follow current guidelines [20, 23]. This contrasts with testing for $A P C$ or $B R C A 1 / 2$ variants, where geneticists have a crucial part to play in the clinical pathway (i.e. patient referral, diagnosis and counselling), even though clinicians guide the care management. The role of primary care physicians in making medical decisions that concern genetic investigations remains very marginal, as confirmed in other studies [26, 27].

Almost no participants referred to clinical or psychological harms resulting from the genetic information obtained from testing. The direction of our findings on the quality of life showed that patients who underwent $A P C$ or $B R C A 1 / 2$ testing were more likely to indicate improvements in their daily life than those tested for thrombophilia. As reported in other studies, individuals undergoing DNA testing per se (e.g. thrombophilia testing) do not perceive such an experience as exceptional or unusual; indeed, the majority of patients do not even recall having had a genetic test [23, 28]. Consequently, they do not have strong opinions on any consequent improvement in their quality of 
life. In our study, a large proportion of participants undergoing thrombophilia screening asked interviewers for further clarification of the term 'quality of life', as opposed to patients who were tested for cancer-related diseases $(\mathrm{Q} 8$, Table 2). Some authors have argued that such requests for further information by patients is because they are being tested for very low-risk genetic susceptibilities, which have little or no impact on their daily activities or health-care management $[23,28]$. Thus, answers relating to the quality of life could be indirectly affected by the overall utility of genetic testing for a given condition.

Another finding of our study is that, alongside the type of testing, carrier status can influence the self-perceived quality of life, i.e. non-carriers reported a better quality of life than carriers. This is in line with results obtained by other studies that analysed carrier status as a potential determinant of patient experience outcomes [29-36]. Carrying a diseasepredisposing variant may cause problems for patients seeking insurance (depending on the health-care context); patients may also experience stigmatization and anxiety for their future health, all of which can be assumed to affect quality of life [29-36].

The current study is the first original research to analyse a potential association between patient experience outcomes and type of testing as main predictor, distinguishing between genetic tests with determined or undetermined utility. A qualitative review by Meiser et al. [37] limited the analysis of patient experience to cancer susceptibility testing (i.e. hereditary breast and ovarian cancer, familial adenomatous polyposis, and hereditary non-polyposis colorectal cancer), concluding that the psychological impact of DNA testing is partly a reflection of the clarity of the risk management options associated with the genetic test undertaken. Our research included a genetic susceptibility test, i.e. that for thrombophilia, which provided little or no clarity on risk management, highlighting the issue described by Meiser et al. [37]. Moreover, ours is one of only a few studies that investigate patient outcomes over the mediumto long term. Most of the previous literature is concerned with psychological outcomes over a short period after patients received their test results (a few weeks or months later), while the present study undertook to obtain a better grasp of the perception of quality of life by assessing patient outcomes a mean of 3 years after testing took place.

This study has several limitations, the first concerning the measurement of quality of life. Because a clear need to slim down the questionnaire emerged in the pilot study, we did not administer a validated questionnaire to estimate the quality of life, but instead relied on direct questions. Interviewers explained to patients the meaning of quality of life, including any harms they experienced, using a brief summary based on questions reported in the WHO Quality of Life-BREF [38]. Second, the long time period between testing and interviews could result in recall bias, especially concerning satisfaction with genetic counselling. Another limitation is that, while we controlled for several potential confounders, there could be other factors that are associated with both the outcomes of patient experience and the tests selected for the study (e.g., risk of a cancerous disease, severe changing of lifestyle due to prophylactic surgeries, or misuse of thrombophilia test due to over-prescription). These unmeasured confounders should be taken into account when interpreting the results. A final limitation is the generalizability of the study, in view of the fact that the research was conducted in only one centre. However, the genetic service of San Camillo-Forlanini Hospital provides a reference genetic service and receives a huge number of patient referrals from all over Italy. The study is therefore likely to be representative of the broader Italian population.

\section{Conclusions}

Assessment of patient experience helps to define the performance of a genetic service. Our results show that patient experience depends, at least partially, on the type of genetic test carried out, with the overall utility of the test apparently being an important factor. In line with international recommendations, genetic tests should be offered only once their utility has been demonstrated, as in the case of $A P C$ and $B R C A 1 / 2$ testing. Successful models for functional integration of genetics with other clinical specialties can improve patient experience, reducing inappropriate referrals.

\section{Compliance with ethical standards}

Conflict of interest Part of this work is connected to the project "L'impatto economico dei test genetici sul Servizio Sanitario Nazionale (SSN): valutazione dei percorsi diagnostico-assistenziali, stime di costoefficacia e costo-utilità e analisi delle politiche sanitarie a livello europeo" [The economic impact of genetic testing on the National Health Service: evaluation of diagnostic care pathways, estimates of cost-effectiveness and cost-utility, and investigation of health policies in Europe], funded by the Italian Ministry of Health; and to the project "Personalized pREvention of Chronic DIseases consortium (PRECeDI)" funded by the European Research and Innovation programme Horizon 2020, under grant agreement no. 645740 . All authors have taken part in both projects.

Open Access This article is licensed under a Creative Commons Attribution-NonCommercial-NoDerivatives 4.0 International License, which permits any non-commercial use, sharing, distribution and reproduction in any medium or format, as long as you give appropriate credit to the original author(s) and the source, and provide a link to the Creative Commons license. You do not have permission under this license to share adapted material derived from this article or parts of it. The images or other third party material in this article are included in the article's Creative Commons license, unless indicated otherwise in a credit line to the material. If material is not included in the article's Creative Commons license and your intended use is not permitted by statutory regulation or exceeds the permitted use, you will need to obtain permission directly from the copyright holder. To view a copy of this license, visit http://creativecommons.org/licenses/by-nc-nd/4.0/. 


\section{References}

1. Mazzucco W, Pastorino R, Lagerberg T,et al. Current state of genomic policies in healthcare among EU member states: results of a survey of chief medical officers. Eur J Public Health. 2016;27:931-7.

2. Payne K, Nicholls S, McAllister M, Macleod R, Donnai D, Davies LM. Outcome measurement in clinical genetics services: a systematic review of validated measures. Value Health. 2008;11:497-508.

3. Chatterjee P, Tsai TC, Jha AK. Delivering value by focusing on patient experience. Am J Manag Care. 2015;21:735-7.

4. Isaac T, Zaslavsky AM, Cleary PD, Landon BE. The relationship between patients' perception of care and measures of hospital quality and safety. Health Serv Res. 2010;45:1024-40.

5. Anhang PR, Elliott MN, Zaslavsky AM, et al. Examining the role of patient experience surveys in measuring health care quality. Med Care Res Rev. 2014;71:522-54.

6. Bleich SN, Ozaltin E, Murray CK. How does satisfaction with the health-care system relate to patient experience? Bull World Health Organ. 2009;87:271-8.

7. Grosse SD, Khoury MJ. What is the clinical utility of genetic testing? Genet Med. 2006;8:448-50.

8. Pitini E, De Vito C, Marzuillo C, et al. How is genetic testing evaluated? A systematic review of the literature. Submitted to Eur J Hum Genet. 2017.

9. Kohler JN, Turbitt E, Biesecker BB. Personal utility in genomic testing: a systematic literature review. Eur J Hum Genet. 2017;25:662-8.

10. Aretz S, Vasen HFA, Olschwang S. Clinical Utility Gene Card for: familial adenomatous polyposis (FAP) and attenuated FAP (AFAP) - update 2014. Eur J Hum Genet. 2015;23:890.

11. Grosse SD, Kalman L, Khoury MJ. Evaluation of the validity and utility of genetic testing for rare diseases. Adv Exp Med Biol. 2010;686:115-31.

12. Kudo M, Lee HL, Yang IA, Masel PJ. Utility of thrombophilia testing in patients with venous thrombo-embolism. J Thorac Dis. 2016;8:3697-703.

13. Bursac Z, Gauss CH, Williams DK, Hosmer DW. Purposeful selection of variables in logistic regression. Source Code Biol Med. 2008;3:17.

14. Allison PD. Convergence failures in logistic regression. In: Proceedings of the SAS Global Forum 2008 Conference. Cary, NC: SAS Institute Inc; 2008. p. 1-11.

15. D'Andrea E, Marzuillo C, De Vito C, et al. Which BRCA genetic testing programs are ready for implementation in health care? A systematic review of economic evaluations. Genet Med. 2016;18:1171-80.

16. D'Andrea E, Marzuillo C, Pelone F, De Vito C, Villari P. Genetic testing and economic evaluations: a systematic review of the literature. Epidemiol Prev. 2015;39(4 Suppl 1):45-50.

17. Stevens SM, Woller SC, Bauer KA, et al. Guidance for the evaluation and treatment of hereditary and acquired thrombophilia. $\mathbf{J}$ Thromb Thrombolysis. 2016;41:154-64.

18. Bates SM, Greer IA, Middeldorp S,et al. VTE, thrombophilia, antithrombotic therapy, and pregnancy: antithrombotic therapy and prevention of thrombosis, 9th ed: ACCP Ev. based clinical practice guidelines. Chest. 2012;141(2 Suppl):e691S-e736S.

19. Ashida S, Koehly LM, Roberts JS, Chen CA, Hiraki S, Green RC. Disclosing the disclosure: factors associated with communicating the results of genetic susceptibility testing for Alzheimer's disease. J Health Commun. 2009;14:768-84.

20. Rolland JS, Williams JK. Toward a biopsychosocial model for 21st-century genetics. Fam Process. 2005;44:3-24.

21. Middeldorp S, van Hylckama Vlieg A. Does thrombophilia testing help in the clinical management of patients? $\mathrm{Br} \mathrm{J}$ Haematol. 2008;143:321-35.

22. Merriman L, Greaves M. Testing for thrombophilia: an evidencebased approach. Postgrad Med J. 2006;82:699-704.

23. Saukko P, Richards SH, Shepherd MH, John JL. Are genetic tests exceptional? Lessons from a qualitative study on thrombophilia. Soc Sci Med. 2006;63:1947-59.

24. PHG Foundation. Genetics and mainstream medicine: service development and integration. Cambridge: PHG Foundation; 2011.

25. Marzuillo C, De Vito C, D'Addario $M$, et al. Are public health professionals prepared for public health genomics? A cross-sectional survey in Italy. BMC Health Serv Res. 2014;14:239.

26. Marzuillo C, De Vito C, Boccia S, et al. Knowledge, attitudes and behavior of physicians regarding predictive genetic tests for breast and colorectal cancer. Prev Med. 2013;57:477-82.

27. Bellcross CA, Kolor K, Goddard KA, et al. Awareness and utilization of BRCA1/2 testing among US primary care physicians. Am J Prev Med. 2011;40:61-6.

28. Hallowell N, Jenkins N, Douglas M, et al. A qualitative study of patients' perceptions of the value of molecular diagnosis for familial hypercholesterolemia $(\mathrm{FH}) . \mathrm{J}$ Comm Genet. 2017;8:45-52.

29. Macrae L, de Souza AN, Loiselle CG, Wong N. Experience of BRCA1/2 mutation-negative young women from families with hereditary breast and ovarian cancer: a qualitative study. Hered Cancer Clin Pract. 2013;11:14.

30. Metcalfe K, Poll A, Llacuachaqui M, et al. Patient satisfaction and cancer-related distress among unselected Jewish women undergoing genetic testing for BRCA1 and BRCA2. Clin Genet. 2010;78:411-7.

31. Brunstrom K, Murray A, McAllister M. Experiences of women who underwent predictive BRCA1/2 mutation testing before the age of 30. J Genet Couns. 2015;25:90-100.

32. Foster C, Watson M, Eeles R, et al. Predictive genetic testing for BRCA1/2 in a UK clinical cohort: three-year follow-up. Br J Cancer. 2007;96:718-24.

33. Low CA, Bower JE, Kwan L, Seldon J. Benefit finding in response to BRCA1/2 testing. Ann Behav Med. 2008;35: 61-9.

34. Cohn DM, Vansenne F, Kaptein AA, De Borgie CA, Middeldorp $\mathrm{S}$. The psychological impact of testing for thrombophilia: a systematic review. J Thromb Haemost. 2008;6:1099-104.

35. Douma KF, Aaronson NK, Vasen HF, et al. Psychological distress and use of psychosocial support in familial adenomatous polyposis. Psychooncology. 2010;19:289-98.

36. Codori AM, Zawacki KL, Petersen GM, et al. Genetic testing for hereditary colorectal cancer in children: long-term psychological effects. Am J Med Genet A. 2003;116:117-28.

37. Meiser B. Psychological impact of genetic testing for cancer susceptibility: an update of the literature. Psychooncology. 2005; 14:1060-74.

38. The Whoqol Group. The World Health Organization quality of life assessment (WHOQOL): Development and general psychometric properties. Soc Sci Med. 1998;46:1569-8. 